\title{
Document Version Text
}

National Cancer Institute

\section{Source}

National Cancer Institute. Document Version Text. NCI Thesaurus. Code C93820.

A textual or media-based representation that is the full or comprehensive narrative or content of the document. 